\title{
THE TRENDS HIGH-CONTRAST IMAGING SURVEY. II. DIRECT DETECTION OF THE HD 8375 TERTIARY
}

\author{
Justin R. Crepp ${ }^{1}$, John Asher Johnson ${ }^{2,3}$, Andrew W. Howard ${ }^{4}$, Geoff W. Marcy ${ }^{5}$, Debra A. Fischer ${ }^{6}$, \\ SCOTT M. YANTEK ${ }^{2}$, JASON T. WRIGHT ${ }^{7,8}$, HOWARD ISAACSON ${ }^{5}$, AND YING FENG ${ }^{7,8}$ \\ ${ }^{1}$ Department of Physics, University of Notre Dame, 225 Nieuwland Science Hall, Notre Dame, IN 46556, USA; jcrepp@nd.edu \\ 2 Department of Astronomy, California Institute of Technology, 1200 E. California Blvd., Pasadena, CA 91125, USA \\ ${ }^{3}$ NASA Exoplanet Science Institute (NExScI), CIT Mail Code 100-22, 779 South Wilson Avenue, Pasadena, CA 91125, USA \\ ${ }^{4}$ Institute for Astronomy, University of Hawaii, 2680 Woodlawn Drive, Honolulu, HI 96822, USA \\ ${ }^{5}$ Department of Astronomy, University of California, Berkeley, CA 94720, USA \\ ${ }^{6}$ Department of Physics, Yale University, New Haven, CT 06511, USA \\ ${ }^{7}$ Department of Astronomy \& Astrophysics, The Pennsylvania State University, University Park, PA 16802, USA \\ ${ }^{8}$ Center for Exoplanets and Habitable Worlds, The Pennsylvania State University, University Park, PA 16802, USA \\ Received 2012 October 27; accepted 2013 May 10; published 2013 June 14
}

\begin{abstract}
We present the direct imaging detection of a faint tertiary companion to the single-lined spectroscopic binary HD $8375 \mathrm{AB}$. Initially noticed as an $53 \mathrm{~m} \mathrm{~s}^{-1} \mathrm{yr}^{-1}$ Doppler acceleration by Bowler et al., we have obtained high-contrast adaptive optics observations at Keck using NIRC2 that spatially resolve HD $8375 \mathrm{C}$ from its host(s). Astrometric measurements demonstrate that the companion shares a common proper-motion. We detect orbital motion in a clockwise direction. Multiband relative photometry measurements are consistent with an early M-dwarf spectral type $(\sim \mathrm{M} 1 \mathrm{~V})$. Our combined Doppler and imaging observations place a lower-limit of $m \geqslant 0.297 M_{\odot}$ on its dynamical mass. We also provide a refined orbit for the inner pair using recent radial velocity measurements obtained with the High Resolution Echelle Spectrometer. HD 8375 is one of many triple-star systems that are apparently missing in the solar neighborhood.
\end{abstract}

Key words: astrometry - brown dwarfs - methods: observational - stars: individual (HD 8375) - stars: low-mass - techniques: high angular resolution - techniques: radial velocities

Online-only material: color figure

\section{INTRODUCTION}

We have recently commenced an interdisciplinary program that combines the radial velocity (RV) method with highcontrast imaging to identify faint companions in wide orbits around nearby stars (Crepp et al. 2012b). Using approximately $7-25 \mathrm{yr}$ of precise Doppler measurements, we select targets for follow-up adaptive optics (AO) observations based on the existence of long-term RV accelerations (trends), which indicate the presence of a distant body. The objective of the TaRgetting bENchmark objects with Doppler Spectroscopy (TRENDS) high-contrast imaging program is to directly detect and study in detail low-temperature bodies that are found to orbit each target star.

Once a companion is identified, its relative position on the sky is measured over multiple epochs with imaging, and the primary star is monitored at increased cadence with continued Doppler measurements. Together, these observations can determine the three-dimensional orbit and precise dynamical mass of the companion (Crepp et al. 2012a). Spectro-photometric measurements acquired across a wide bandpass may then be used to test theoretical spectral models and thermal evolutionary models by comparing the inferred mass to that found using Newton's laws (Boden et al. 2006). The ultimate goal of the TRENDS program is to determine the first orbit (all six elements) and dynamical mass of a directly imaged gas giant extrasolar planet.

The majority of our targets are nearby $(d \lesssim 100$ pc), bright $(V \lesssim 12)$, main-sequence FGK stars with RV time-baselines of at least several years. We also monitor M-dwarfs (Apps et al. 2010), massive subgiants (Johnson et al. 2011), and young stars (L. A. Hillenbrand et al., in preparation). In many cases, Doppler measurements span more than a decade (e.g., Wright et al. 2009).
A number of systems already show curvature (change in the RV acceleration), making it possible to characterize the orbit rapidly and more accurately than astrometry-only surveys (Konopacky et al. 2010; Dupuy et al. 2010).

The TRENDS survey began observations of an intrinsically companion-rich sample of stars in 2010 May at Keck using NIRC2 (Crepp et al. 2012b). Many targets have a large proper-motion, and follow-up observations demonstrate that their companions are comoving. In this paper, we report the direct detection of HD $8375 \mathrm{C}$, the tertiary companion of a single-lined spectroscopic binary. A Doppler acceleration of $53 \mathrm{~m} \mathrm{~s}^{-1} \mathrm{yr}^{-1}$ was initially noticed by Bowler et al. (2010) over a $5.3 \mathrm{yr}$ baseline. We show that HD $8375 \mathrm{C}$ is responsible for the trend. Our recent RV measurements improve the orbit solution for HD $8375 \mathrm{AB}$ and extend the baseline of the observed acceleration.

It is known that the census of triple-stars is currently incomplete beyond $10 \mathrm{pc}$ (Tokovinin 2004). At a distance of $56.7 \pm 1.3 \mathrm{pc}, \mathrm{HD} 8375$ (Table 1) represents one of many hierarchical systems that are apparently missing in the solar neighborhood, having thus far evaded detection by various faintcompanion search techniques. Furthermore, few triple systems have had their orbital dynamics studied in detail (Duchêne et al. 2006; Schaefer et al. 2012). Also, determining the configuration of multi-star systems has important implications for our understanding of star-formation theory, which is constrained primarily from observations of single and binary stars (Crepp et al. 2010; Bate 2009). Given the benefits of combining two complementary techniques, such as precise Doppler measurements and direct imaging, the HD 8375 system provides an excellent opportunity to characterize a hierarchical triple-star system in exquisite detail. 


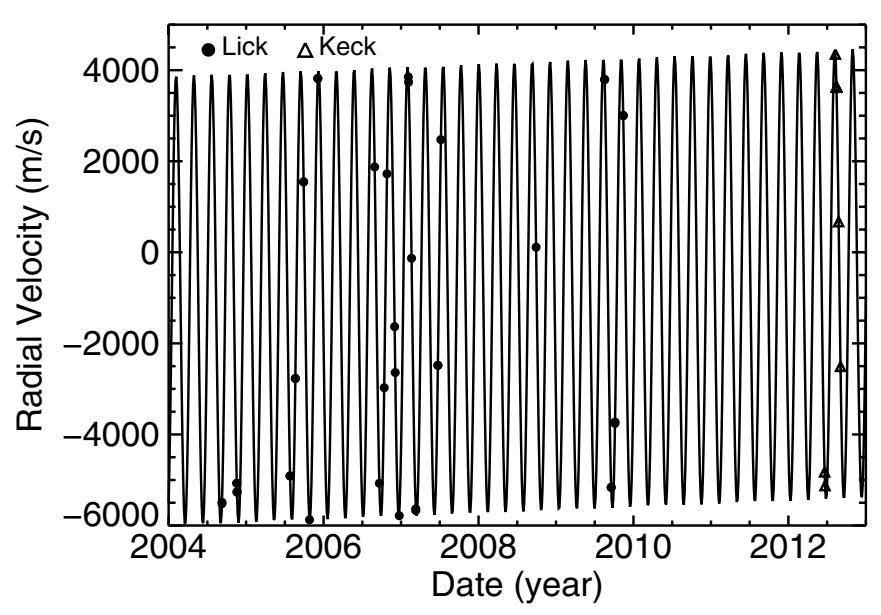

Figure 1. Precise Doppler measurements of HD 8375. An SB1 stellar pair with an 84 day period exhibits a subtle long-term acceleration of $67.4 \pm$ $2.2 \mathrm{~m} \mathrm{~s}^{-1} \mathrm{yr}^{-1}$, suggesting the presence of a distant tertiary companion.

Table 1

Coordinates, Apparent Magnitudes, Distance, and Proper Motion of HD 8375 from SIMBAD

\begin{tabular}{lc}
\hline \multicolumn{2}{c}{ HD 8375 System Properties } \\
\hline Right ascension (J2000) & 012337.5 \\
Declination (J2000) & +341445.2 \\
$B$ & 7.1 \\
$V$ & 6.3 \\
$J$ & $4.820 \pm 0.037$ \\
$H$ & $4.222 \pm 0.236$ \\
$K_{s}$ & $4.290 \pm 0.023$ \\
$d($ pc) & $56.7 \pm 1.3$ \\
Proper motion $\left(\right.$ mas yr $\left.^{-1}\right)$ & $233.1 \pm 0.3 \mathrm{E}$ \\
& $117.8 \pm 0.2 \mathrm{~N}$ \\
\hline
\end{tabular}

Notes. Magnitudes are from 2MASS (Skrutskie et al. 2006). The parallax-based distance is from Hipparcos measurements using the refined data reduction of van Leeuwen (2007).

\section{OBSERVATIONS}

\subsection{Doppler Measurements}

HD 8375 was initially noticed as an (SB1) RV variable star by Beavers \& Eitter (1986). Also identified as an evolved star by de Medeiros \& Mayor (1999), and Snowden \& Young (2005), HD 8375 AB has a designated spectral type of G8IV. Most recently, it was targeted by Johnson et al. (2006) at Lick Observatory as part of a dedicated Doppler survey of subgiants to search for extrasolar planets. From this data set the first accurate orbit for $\mathrm{HD} 8375 \mathrm{AB}$ was published by Bowler et al. (2010). In addition to a large SB1 signal $\left(K=4939.2_{-2.5}^{+2.6} \mathrm{~m} \mathrm{~s}^{-1}\right)$, a subtle long-term acceleration, $d v / d t=52.9_{-1.9}^{+1.8} \mathrm{~m} \mathrm{~s}^{-1} \mathrm{yr}^{-1}$, suggested that the HD $8375 \mathrm{AB}$ pair is orbited by a tertiary companion.

The Johnson et al. (2006) survey measurements were obtained with the Hamilton echelle spectrometer (Vogt 1987) at Lick Observatory using the $3 \mathrm{~m}$ Shane Telescope and $0.6 \mathrm{~m}$ Coudé Auxiliary Telescope. The first observations of HD 8375 began on 2004 September 8 (Table 2). Subsequent to the identification of a long-term trend, we also began Doppler monitoring with the HIgh Resolution Echelle Spectrometer (HIRES; Vogt et al. 1994) at Keck (Table 3). Figure 1 shows the relative RV measurements used for this study, which span $8.0 \mathrm{yr}$ and include the Bowler et al. (2010) data points along with seven recent measurements from HIRES.
Table 2

Lick RV Measurements

\begin{tabular}{lrc}
\hline \hline HJD-2,450,000 & $\begin{array}{c}\text { RV } \\
\left(\mathrm{m} \mathrm{s}^{-1}\right)\end{array}$ & $\begin{array}{c}\text { Uncertainty } \\
\left(\mathrm{m} \mathrm{s}^{-1}\right)\end{array}$ \\
\hline 3256.962 & -3807.92 & 6.67 \\
3256.984 & -3778.30 & 6.48 \\
3326.717 & -3359.07 & 5.39 \\
3326.737 & -3360.36 & 6.14 \\
3327.756 & -3551.16 & 6.72 \\
3327.778 & -3553.56 & 6.91 \\
3577.974 & -3199.62 & 8.29 \\
3577.994 & -3196.42 & 7.06 \\
3602.865 & -1058.33 & 5.53 \\
3602.888 & -1058.29 & 5.52 \\
3641.865 & 3260.39 & 5.07 \\
3641.882 & 3256.72 & 5.24 \\
3669.696 & -4161.93 & 8.64 \\
3708.725 & 5528.20 & 6.24 \\
3708.750 & 5530.83 & 5.49 \\
3976.821 & 3586.67 & 5.38 \\
3998.874 & -3360.45 & 6.62 \\
4021.860 & -1261.40 & 9.15 \\
4034.861 & 3436.29 & 7.47 \\
4070.777 & 78.87 & 6.61 \\
4073.672 & -928.42 & 5.02 \\
4092.661 & -4071.47 & 10.18 \\
4135.638 & 5562.40 & 4.33 \\
4136.638 & 5448.56 & 4.45 \\
4150.645 & 1581.30 & 5.54 \\
4170.617 & -3921.77 & 6.86 \\
4170.633 & -3957.81 & 7.71 \\
4274.979 & -769.69 & 5.91 \\
4274.996 & -770.38 & 7.26 \\
4288.965 & 4186.74 & 5.55 \\
4737.842 & 1824.03 & 6.16 \\
5060.954 & 5510.28 & 6.20 \\
5060.968 & 5501.75 & 6.86 \\
5091.868 & -3448.10 & 6.65 \\
5091.881 & -3452.50 & 6.45 \\
5109.840 & -2046.18 & 7.71 \\
5109.873 & -2015.68 & 7.99 \\
5148.778 & 4712.08 & 7.42 \\
5148.794 & 4717.75 & \\
\hline & & \\
& & \\
& & \\
& &
\end{tabular}

Table 3

Keck HIRES RV Measurements

\begin{tabular}{lcc}
\hline \hline HJD-2,450,000 & $\begin{array}{c}\text { RV } \\
\left(\mathrm{m} \mathrm{s}^{-1}\right)\end{array}$ & $\begin{array}{c}\text { Uncertainty } \\
\left(\mathrm{m} \mathrm{s}^{-1}\right)\end{array}$ \\
\hline 6098.133 & -4823.01 & 1.37 \\
6100.109 & -5124.58 & 1.37 \\
6149.026 & 4351.28 & 1.08 \\
6154.045 & 3643.48 & 1.03 \\
6154.140 & 3623.26 & 1.04 \\
6164.119 & 671.85 & 0.94 \\
6173.095 & -2506.32 & 1.18 \\
\hline
\end{tabular}

The additional RV measurements from HIRES allow us to refine the orbital solution of HD 8375 AB. Following the methodology of Wright \& Howard (2009), we use the RVLIN software package ${ }^{9}$ to simultaneously fit the Lick and Keck velocities. Parameter uncertainties are derived following a "boot-strap analysis" methodology described in Wang et al. (2012) with the boottran software package. For this fit we

\footnotetext{
9 RVLIN and boottran are available at http://exoplanets.org/code
} 
Table 4

Physical Properties of HD 8375 A and Refined Orbital Parameters for HD 8375 AB

\begin{tabular}{lc}
\hline \multicolumn{2}{c}{ HD 8375 A } \\
\hline Mass $\left(M_{\odot}\right)$ & $1.45 \pm 0.12$ \\
Radius $\left(R_{\odot}\right)$ & $3.7 \pm 0.2$ \\
Luminosity $\left(L_{\odot}\right)$ & $8.5 \pm 0.5$ \\
{$[\mathrm{Fe} / \mathrm{H}]$} & $-0.13 \pm 0.04$ \\
$\log g\left(\mathrm{~cm} \mathrm{~s}^{-2}\right)$ & $3.46 \pm 0.06$ \\
$T_{\text {eff }}(\mathrm{K})$ & $5103 \pm 44$ \\
Spectral type & $\mathrm{G} 8 \mathrm{IV}$ \\
$v \sin i\left(\mathrm{~km} \mathrm{~s}^{-1}\right)$ & $2.0 \pm 0.5$ \\
\hline & \\
\hline$m_{B} \sin (i)\left(M_{\odot}\right)$ & \\
$P(\mathrm{days})$ & $0.137 \pm 0.007$ \\
$K\left(\mathrm{~m} \mathrm{~s}^{-1}\right)$ & $83.9428 \pm 0.0013$ \\
$e$ & $4931.0 \pm 3.0$ \\
$\omega\left(^{\circ}\right)$ & $0.0179 \pm 0.0008$ \\
$t_{p}(\mathrm{JD}-2,450,000)$ & $329.50 \pm 2.13$ \\
$\gamma\left(\mathrm{m} \mathrm{s}^{-1}\right)$ & $4125.01 \pm 0.50$ \\
$d v / d t\left(\mathrm{~m} \mathrm{~s}^{-1} \mathrm{yr}^{-1}\right)$ & $-960.4 \pm 14.0$ \\
\hline
\end{tabular}

Notes. Top: physical properties of HD 8375 A derived from SME and theoretical isochrones using HIRES template spectra (Valenti \& Fischer 2005). We notice no contamination in the $\lambda=0.50-0.62 \mu \mathrm{m}$ range from HD $8375 \mathrm{BC}$. Bottom: refined orbital parameters for HD 8375 AB using recent Doppler measurements. Model variables include the period $(P)$, RV semiamplitude $(K)$, eccentricity $(e)$, argument of periastron $(\omega)$, time of periastron passage $\left(t_{p}\right), \mathrm{RV}$ instrument offset from Lick to $\operatorname{Keck}(\gamma)$, and Doppler acceleration $(d v / d t)$. We calculate the minimum mass of HD $8375 \mathrm{~B}$ from RV measurements using the $1.45 \pm 0.12 M_{\odot}$ estimated mass of HD $8375 \mathrm{~A}$.

assume no jitter and allow for an offset between Lick and Keck velocities. Parameters from our updated orbital solution are shown in Table 4.

The rms to our companion plus trend fit is $25 \mathrm{~m} \mathrm{~s}^{-1}$, considerably higher than internal errors. There is no indication that this star should exhibit such high levels of jitter, nor does a periodogram analysis and manual search for a second, shorter period companion reveal any significant signal in the residuals. The residual scatter could be due to a small amount of flux from HD 8735 B contaminating our spectra and complicating the forward modeling procedure in our precise Doppler analysis. However, visual inspection of stellar template spectra does not reveal any anomalous features. HD 8375 A appears to dominate the spectrum of the entire system. Using a crosscorrelation analysis, we place an upper-limit on the amount of flux contamination in the $\lambda=0.50-0.62 \mu \mathrm{m}$ wavelength range at the $1 \%$ level.

There is a hint of curvature in the Lick velocities (which the Keck velocities cannot constrain because of the offset between the telescopes and short time baseline of that particular data set). The velocities increase by $\approx 40 \mathrm{~m} \mathrm{~s}^{-1}$ from 2007 to 2010, a jump that would normally be highly significant, but may be spurious in this case given the large, unexplained rms scatter about the companion plus trend fit. Continued Doppler monitoring is required to assess the change in slope of the observed acceleration over the next several years.

\subsection{Primary Star Properties}

Since close inspection of HIRES observations reveals virtually no contamination from HD 8375 BC, we are justified in
Table 5

Summary of Astrometric Measurements

\begin{tabular}{lcccc}
\hline \hline $\begin{array}{l}\text { Date } \\
\text { (UT) }\end{array}$ & JD-2,450,000 & $\begin{array}{c}\rho \\
(\mathrm{mas})\end{array}$ & $\begin{array}{c}\text { P.A. } \\
\left({ }^{\circ}\right)\end{array}$ & $\begin{array}{c}\text { Projected Separation } \\
(\mathrm{AU})\end{array}$ \\
\hline 2010 Oct 13 & 5482.94 & $326.0 \pm 4.5$ & $48.9 \pm 0.8$ & $18.5 \pm 0.5$ \\
2011 Aug 31 & 5804.06 & $309.5 \pm 2.8$ & $45.3 \pm 0.4$ & $17.5 \pm 0.5$ \\
2012 Aug 25 & 6165.10 & $285.5 \pm 1.1$ & $39.9 \pm 0.3$ & $16.2 \pm 0.4$ \\
\hline
\end{tabular}

Note. HD $8375 \mathrm{C}$ is moving in a clock-wise direction relative to HD $8375 \mathrm{AB}$ and its projected separation is decreasing rapidly with time.

deriving bulk physical properties of the primary star. Stellar (template) spectra, taken with the iodine gas cell removed from the optical path, were analyzed using the LTE spectral synthesis code Spectroscopy Made Easy (SME) described in Valenti $\&$ Fischer (2005). SME provides an estimate of the stellar effective temperature $\left(T_{\text {eff }}\right)$, surface gravity $(\log g)$, metallicity $([\mathrm{Fe} / \mathrm{H}])$, and projected rotational velocity $(v \sin i)$. Table 4 lists the spectral-type and physical properties of the primary star derived from spectral fitting along with comparison to theoretical isochrones. The SME model yields an excellent fit with a reduced $\chi^{2}$ close to one, independently verifying that very little flux from the secondary and tertiary are contaminating the spectra at visible wavelengths.

\subsection{High-contrast Imaging}

Knowing that HD 8375 must have a distant body orbiting the central binary pair, we acquired high-contrast images of the system with NIRC2 (instrument PI: Keith Matthews) using the Keck II AO system (Wizinowich et al. 2000) on 2010 October 13 UT. First, epoch images were taken using the $H$-band filter. The star (spatially unresolved binary) was placed behind the 300 mas diameter coronagraph spot. We used the angular differential imaging technique to discriminate between residual scattered starlight and companions (Marois et al. 2006).

Figure 2 shows images taken before and after speckle suppression. The companion has a brightness comparable to the static speckle pattern though it can be seen in individual pre-processed frames. Using the locally optimized combination of images algorithm (Lafrenière et al. 2007), and derotating frames to align and stack light from off-axis sources, we are able to identify the candidate located within close vicinity and to the north-east of the primary star(s). Follow up observations were taken on 2011 August 31 UT and 2012 August 25 UT with complementary filters to obtain color information and determine whether the candidate tertiary is associated with the primary. We note that the a priori likelihood that the point source is a false-positive is low, given the small (326 mas) angular separation and location of HD 8375 relative to the galactic plane.

\section{ASTROMETRY}

Our astrometric observations consist of three epochs taken over an $1.9 \mathrm{yr}$ time baseline (Table 5). The proper-motion of HD 8375 is high (see Table 1), allowing us to easily determine whether the companion shares the same space motion as the primary. We measured an accurate angular separation and position angle using the technique described in Crepp et al. (2012a). We first fit Gaussian functions to the stellar and companion point-spread functions to locate their centroids in each frame. We then correct for distortion in the NIRC2 focal plane using publicly available solutions provided by Keck 

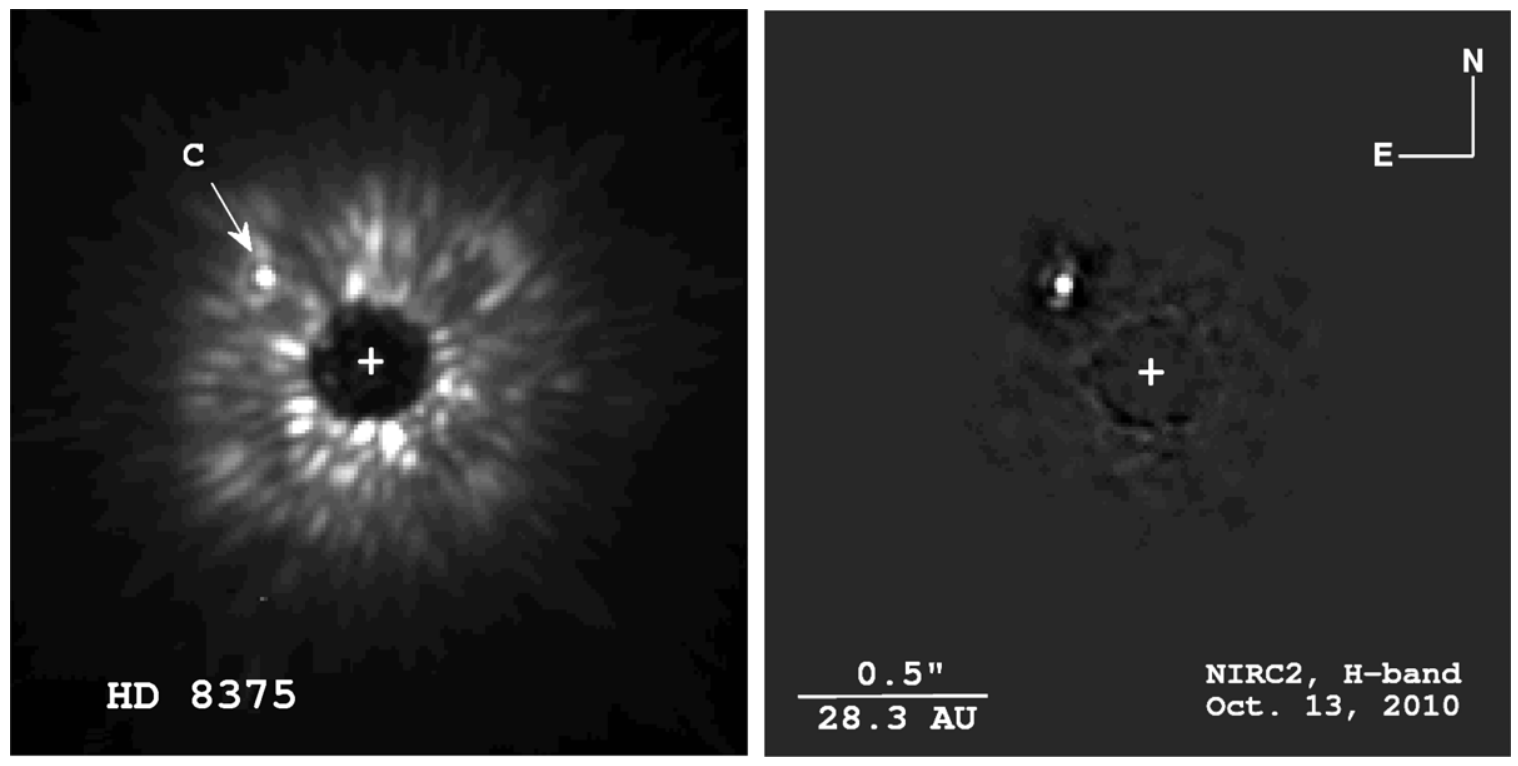

Figure 2. Discovery images of HD $8375 \mathrm{C}$ taken in the $H$ band at Keck with NIRC2 on 2010 October 13 UT. The companion, discernible in individual frames (left), is unambiguously recovered following PSF subtraction (right). A cross denotes the location of the primary star behind the coronagraph.

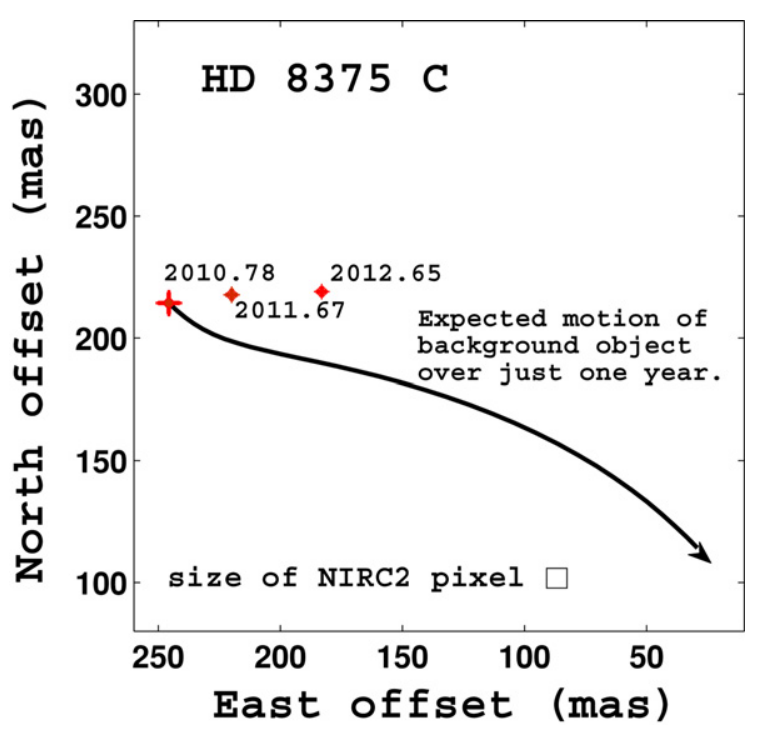

Figure 3. Astrometric measurements (red-crosses) demonstrating that $\mathrm{HD} 8375 \mathrm{C}$ is co-moving with $\mathrm{HD} 8375 \mathrm{AB}$. Axes correspond to the measured angular separation (offset) of HD $8375 \mathrm{C}$ from HD $8375 \mathrm{AB}$. Solid curves show the path that a distant background object with zero proper-motion would follow from 2010 October 13 through 2011 August 31 accounting for stellar proper-motion and parallactic motion. Our measurements span a time frame twice as long, from 2010 October 13 through 2012 August 25; we have zoomed in to show the size of astrometric uncertainties.

(A color version of this figure is available in the online journal.)

Observatory's astrometry support page (Ghez et al. 2008). ${ }^{10}$ The results are averaged and the uncertainty in the separation and position angle is taken as the standard deviation, taking into account uncertainty in the plate scale and orientation of the array by propagating these errors to the final calculated position.

Figure 3 shows multi-epoch astrometry measurements plotted against the expected motion of a distant background object. We find that HD $8375 \mathrm{C}$ is clearly associated with HD $8375 \mathrm{AB}$. The tertiary has a projected separation of $16.2 \pm 0.4 \mathrm{AU}$ as of 2012 August, and appears to exhibit orbital motion in a clock-wise

\footnotetext{
10 http://www2.keck.hawaii.edu/inst/nirc2/dewarp.html
}

Table 6

Tertiary Properties

\begin{tabular}{lc}
\hline \hline \multicolumn{1}{c}{ HD 8375 C } \\
\hline$\Delta J$ & $>4.85$ \\
$\Delta H$ & $5.35 \pm 0.15$ \\
$\Delta K_{s}$ & $4.88 \pm 0.05$ \\
$J$ & $>9.67$ \\
$H$ & $9.56 \pm 0.28$ \\
$K_{s}$ & $9.17 \pm 0.06$ \\
$M_{J}$ & $>5.90$ \\
$M_{H}$ & $5.78 \pm 0.29$ \\
$M_{K_{s}}$ & $5.40 \pm 0.08$ \\
Spectral type & $\approx \mathrm{M} 1 \mathrm{~V}$ \\
$m_{\text {dyn }}\left(M_{\odot}\right)$ & $>0.297$ \\
$m_{\text {model }}\left(M_{\odot}\right)$ & $0.522 \pm 0.039(H$-band $)$ \\
& $0.549 \pm 0.012\left(K_{s}\right.$-band $)$ \\
$m_{\text {empirical }}\left(M_{\odot}\right)$ & $0.44 \pm 0.11(H$-band $)$ \\
& $0.58 \pm 0.04\left(K_{s}\right.$-band $)$
\end{tabular}

Notes. Tertiary companion magnitude difference, apparent magnitude, absolute magnitude, estimated spectraltype, mass constraint from dynamics $\left(m_{\mathrm{dyn}}\right)$, and estimated mass from: (1) photometry using the Dotter et al. (2008) theoretical atmospheric models ( $\left.m_{\text {model }}\right)$, and (2) empirical $M_{H}$-mass and $M_{K}$-mass relations from Delfosse et al. (2000) ( $\left.m_{\text {empirical }}\right)$. Measurements are made relative to the combined (spatially unresolved) light from $\mathrm{HD} 8375 \mathrm{AB}$. The tertiary spectral-type is estimated based on its $H-K_{s}$ color.

direction (north up, east left) as indicated by a slow systematic change in the separation and position angle.

\section{COMPANION MASS}

We estimate the companion mass by comparing its brightness to: (1) late-type dwarfs using Dotter et al. (2008) theoretical evolutionary tracks (the Dartmouth models), (2) empirical relations from Delfosse et al. (2000) that correlate absolute magnitudes with dynamical masses, and (3) our lower-limit constraint on the mass from orbital dynamics. Differential magnitudes, apparent magnitudes, and absolute magnitudes are listed in Table 6. 
We find that HD $8375 \mathrm{C}$ has a mass of $0.522 \pm 0.039 M_{\odot}$ and $0.549 \pm 0.012 M_{\odot}$ based on $H$-band and $K_{s}$-band photometry, respectively (Dotter et al. 2008). These values take into consideration the metallicity of HD $8375 \mathrm{~A}$ (assuming each star has the same chemical composition), and are consistent to within $1 \sigma$. Using Table 5 from Kraus \& Hillenbrand (2007), HD 8375 C has colors and brightness most consistent with an M1-dwarf.

Empirical mass-luminosity relations have a relatively small scatter for M-dwarf stars, often yielding mass predictions accurate to $10 \%$. Substituting absolute magnitudes from Table 6 into the polynomial-fit relations derived by Delfosse et al. 2000, we find that HD $8375 \mathrm{C}$ has a mass of $0.44 \pm 0.11 M_{\odot}$ and $0.58 \pm 0.04 M_{\odot}$ for the $H$ and $K_{s}$ filters, respectively. These values are also consistent with one another and the Dotter et al. (2008) theoretical evolutionary models at the $1 \sigma$ level. We find that this consistency is only true when it is assumed that the tertiary shares the same metallicity as the primary. Invoking a solar metallicity, for instance, introduces an $>1 \sigma$ discrepancy between the mass obtained from empirical relations versus atmospheric models. One can interpret this result as suggestive of a common formation origin, however, the evidence is indirect.

We can place a lower-limit on the companion dynamical mass by combining the RV trend with our measurements of HD 8375 C's projected separation (Torres 1999; Liu et al. 2002). Using our updated Doppler acceleration, $d v / d t=$ $67.4 \pm 2.2 \mathrm{~m} \mathrm{~s}^{-1} \mathrm{yr}^{-1}$, and first epoch direct astrometry from 2010 October, we find that HD $8375 \mathrm{C}$ has a minimum dynamical mass of $m_{\mathrm{dyn}}=0.319 \pm 0.022 M_{\odot}$. This value accounts for uncertainty in the stellar parallax, measured angular separation, and RV acceleration. We thus adopt a minimum mass of $m_{\text {dyn }}=0.297 M_{\odot}$. This lower-limit is consistent with masses derived above from photometry and empirical inference (Table 6). Subsequent observations will further constrain the mass (placing an upper-limit as well) when the HD $8375 \mathrm{C}$ astrometry shows significant curvature.

We can also perform a complementary analysis and selfconsistency check to estimate the true (physical) separation of $\mathrm{HD} 8375 \mathrm{C}$ from (the center of mass of) HD $8375 \mathrm{AB}$ (cf. Howard et al. 2010). If HD $8375 \mathrm{C}$ has a mass of $m_{\text {model }}=$ $0.547 \pm 0.011 M_{\odot}$, which is the weighted average of our $H$ and $K$ estimates from photometry, then the instantaneous orbital separation of HD $8375 \mathrm{C}$ (2012 August) is $16.5 \pm 0.5 \mathrm{AU}$, only marginally larger than the $16.2 \pm 0.4$ AU projected separation. Note that comparing these values does not yield the orbit inclination.

\section{SUMMARY}

We present the second discovery of the TRENDS highcontrast imaging program. Using multi-epoch NIRC2 AO observations at Keck, we have directly imaged the companion responsible for accelerating $\mathrm{HD} 8375 \mathrm{AB}$, a single-line spectroscopic binary with an 83.9 day period. The tertiary, HD $8375 \mathrm{C}$, is only slightly brighter than residual scattered light from the primary. Our multi-band observations indicate that HD $8375 \mathrm{C}$ has brightness and colors consistent with an $\approx \mathrm{M} 1$ dwarf. Combining imaging observations with precise Doppler measurements, we derive a firm lower-limit of $0.297 M_{\odot}$ for the tertiary. HD $8375 \mathrm{C}$ shows measurable orbital motion in a clockwise direction over a 1.9 yr time baseline.

We find that the estimated mass of HD $8375 \mathrm{C}$ is consistent between evolutionary models and empirical mass-luminosity relations, but only once the metallicity of HD $8375 \mathrm{~A}$ is taken into account (assuming a common chemical composition). A dynamical mass with fractional error $<10 \%$ is possible with continued follow-up observations, and will be sufficient to identify any small systematic errors in M-dwarf theoretical atmospheric models.

Follow-up moderate resolution spectroscopy using an integral-field unit will help verify the spectral-type we have assigned to HD 8375 C. Furthermore, near-infrared spectra of the primary can place strong constraints on the properties of HD $8375 \mathrm{~B}$, which is difficult to detect at visible wavelengths, via a combined light analysis. We have recently acquired lowresolution JH spectra of HD $8375 \mathrm{C}$ using Project 1640 at Palomar (Hinkley et al. 2011; Crepp et al. 2011; Pueyo et al. 2012). These observations and analysis will be presented in a separate paper.

HD 8375 is an exemplar hierarchical triple-star system that was initially characterized as a spectroscopic binary. Compared to single stars and binaries, joint Doppler and imaging observations can address more subtle issues involving the star-formation process, such as angular momentum orientation, mass and separation ratios, and the Kozai mechanism. Triple stars represent approximately $8 \%$ of all stellar systems (Raghavan et al. 2010). Thus, we anticipate that the TRENDS high-contrast survey will uncover additional interesting triple-systems for us to study in detail.

The TRENDS high-contrast science program is supported in part by NASA Origins grant NNX13AB03G. The data presented herein were obtained at the W. M. Keck Observatory, which is operated as a scientific partnership among the California Institute of Technology, the University of California and the National Aeronautics and Space Administration. The Observatory was made possible by the generous financial support of the W.M. Keck Foundation. The Center for Exoplanets and Habitable Worlds is supported by the Pennsylvania State University, the Eberly College of Science, and the Pennsylvania Space Grant Consortium.

\section{REFERENCES}

Apps, K., Clubb, K. I., Fischer, D. A., et al. 2010, PASP, 122, 156 Bate, M. R. 2009, MNRAS, 392, 590

Beavers, W. I., \& Eitter, J. J. 1986, ApJS, 62, 147

Boden, A. F., Torres, G., \& Latham, D. W. 2006, ApJ, 644, 1193

Bowler, B. P., Johnson, J. A., Marcy, G. W., et al. 2010, ApJ, 709, 396

Crepp, J., Serabyn, E., Carson, J., Ge, J., \& Kravchenko, I. 2010, ApJ, 715,1533

Crepp, J. R., Johnson, J. A., Fischer, D. A., et al. 2012a, ApJ, 751, 97

Crepp, J. R., Johnson, J. A., Howard, A. W., et al. 2012b, ApJ, 761, 39

Crepp, J. R., Pueyo, L., Brenner, D., et al. 2011, ApJ, 729, 132

de Medeiros, J. R., \& Mayor, M. 1999, A\&AS, 139, 433

Delfosse, X., Forveille, T., Ségransan, D., et al. 2000, A\&A, 364, 217

Dotter, A., Chaboyer, B., Jevremović, D., et al. 2008, ApJS, 178, 89

Duchêne, G., Beust, H., Adjali, F., Konopacky, Q. M., \& Ghez, A. M. 2006, A\&A, 457, L9

Dupuy, T. J., Liu, M. C., Bowler, B. P., et al. 2010, ApJ, 721, 1725

Ghez, A. M., Salim, S., Weinberg, N. N., et al. 2008, ApJ, 689, 1044

Hinkley, S., Oppenheimer, B. R., Zimmerman, N., et al. 2011, PASP, 123, 74

Howard, A. W., Johnson, J. A., Marcy, G. W., et al. 2010, ApJ, 721, 1467

Johnson, J. A., Clanton, C., Howard, A. W., et al. 2011, ApJS, 197, 26

Johnson, J. A., Marcy, G. W., Fischer, D. A., et al. 2006, ApJ, 652, 1724

Konopacky, Q. M., Ghez, A. M., Barman, T. S., et al. 2010, ApJ, 711, 1087

Kraus, A. L., \& Hillenbrand, L. A. 2007, AJ, 134, 2340

Lafrenière, D., Doyon, R., Marois, C., et al. 2007, ApJ, 670, 1367

Liu, M. C., Fischer, D. A., Graham, J. R., et al. 2002, ApJ, 571, 519

Marois, C., Lafrenière, D., Doyon, R., Macintosh, B., \& Nadeau, D. 2006, ApJ, 641,556

Pueyo, L., Crepp, J. R., Vasisht, G., et al. 2012, ApJS, 199, 6

Raghavan, D., McAlister, H. A., Henry, T. J., et al. 2010, ApJS, 190, 1 
Schaefer, G. H., Prato, L., Simon, M., \& Zavala, R. T. 2012, ApJ, 756, 120

Skrutskie, M. F., Cutri, R. M., Stiening, R., et al. 2006, AJ, 131, 1163

Snowden, M. S., \& Young, A. 2005, ApJS, 157, 126

Tokovinin, A. 2004, RMxAC, 21, 7

Torres, G. 1999, PASP, 111, 169

Valenti, J. A., \& Fischer, D. A. 2005, ApJS, 159, 141 van Leeuwen, F. 2007, A\&A, 474, 653

Vogt, S. S. 1987, PASP, 99, 1214

Vogt, S. S., Allen, S. L., Bigelow, B. C., et al. 1994, Proc. SPIE, 2198, 362

Wang, S. X., Wright, J. T., Cochran, W., et al. 2012, ApJ, 761, 46

Wizinowich, P., Acton, D. S., Shelton, C., et al. 2000, PASP, 112, 315

Wright, J. T., \& Howard, A. W. 2009, ApJS, 182, 205

Wright, J. T., Upadhyay, S., Marcy, G. W., et al. 2009, ApJ, 693, 1084 